\title{
Genome-wide identification of novel intergenic enhancer-like elements: implications in the regulation of transcription in Plasmodium falciparum
}

\author{
Suyog Ubhe ${ }^{1}$ Mukul Rawat ${ }^{1}$, Srikant Verma², Krishanpal Anamika² and Krishanpal Karmodiya ${ }^{*}$
}

\begin{abstract}
Background: The molecular mechanisms of transcriptional regulation are poorly understood in Plasmodium falciparum. In addition, most of the genes in Plasmodium falciparum are transcriptionally poised and only a handful of cis-regulatory elements are known to operate in transcriptional regulation. Here, we employed an epigenetic signature based approach to identify significance of previously uncharacterised intergenic regions enriched with histone modification marks leading to discovery of enhancer-like elements.

Results: We found that enhancer-like elements are significantly enriched with H3K4me1, generate unique noncoding bi-directional RNAs and majority of them can function as cis-regulators. Furthermore, functional enhancer reporter assay demonstrates that the enhancer-like elements regulate transcription of target genes in Plasmodium falciparum. Our study also suggests that the Plasmodium genome segregates functionally related genes into discrete housekeeping and pathogenicity/virulence clusters, presumably for robust transcriptional control of virulence/pathogenicity genes.

Conclusions: This report contributes to the understanding of parasite regulatory genomics by identification of enhancer-like elements, defining their epigenetic and transcriptional features and provides a resource of functional cis-regulatory elements that may give insights into the virulence/pathogenicity of Plasmodium falciparum.
\end{abstract}

Keywords: Enhancer identification, Histone modifications, Transcription, ChIP-sequencing and gene regulation

\section{Background}

Malaria is a life threatening disease caused by a parasite of Plasmodium species from apicomplexan genera, with an estimated 200 million cases every year [1]. Plasmodium falciparum is the most virulent species with complex life-cycle involving two hosts - humans and mosquitoes. The clinical manifestation of malaria is a result of Plasmodium's growth in red blood cells (RBCs), where it progresses through morphologically and developmentally distinct stages namely ring, trophozoite, and schizont to multiply into $16-32$ merozoites to invade fresh RBCs upon bursting. The rapid transition between

\footnotetext{
* Correspondence: krish@iiserpune.ac.in

'Department of Biology, Indian Institute of Science Education and Research, Pashan, Pune 411008, India

Full list of author information is available at the end of the article
}

these morphological states is associated with tight control of gene expression, which is poorly understood in $P$. falciparum.

The haploid genome map of P. falciparum laboratory strain 3D7 was published more than a decade ago. Of the $23 \mathrm{Mb}$ nucleotide genome, 53\% constitutes coding while the remaining $47 \%$ is predicted to be non-coding DNA (ncDNA) [2]. RNA-sequencing data revealed that the gene expression is fine-tuned with the intraerythrocytic cycle (IEC) [3, 4]. Transcription in eukaryotic organisms is exquisitely orchestrated by concerted interplay of transcriptional regulatory elements hence identification of such regulatory DNA elements has been extensively explored. Numerous regulatory elements such as core promoters, $\mathrm{G}$ box, downstream regulatory elements, insulators have been identified while 
some like TATA box, and cis-acting elements have been identified and proposed to play roles in transcriptional regulation, however, some of them need functional validation [5-9]. Also, substantial amount of research lead to identification of Myb1, HMG box proteins, and ApiAp2 group of transcription factors [10-13].

Transcriptional enhancer elements are one such type of non-coding regulatory DNA sequences that can enhance transcription of target genes regardless of their location and orientation relative to their target promoter by virtue of chromatin looping [14]. Due to the difficulties presented by extremely AT-rich genome and with no fixed physical distance between regulatory elements and the genes they regulate, the task of finding enhancer elements in Plasmodium genome has been challenging. To-date only a few enhancer elements are known in Plasmodium and their identification has relied on traditional approaches of assaying for differences in functional reporter activities by truncating the DNA sequences [15-17]. Therefore, identification and functional characterisation of enhancer elements from genome by using non-traditional newer approaches is of particular importance to explore the mechanism of transcriptional regulation imposed in $P$. falciparum.

Recently, we generated a comprehensive epigenome map of $P$. falciparum by performing chromatin immunoprecipitation-sequencing (ChIP-seq) for multiple histone modifications at three different IECdevelopmental stages - ring, trophozoite, and schizont $[18,19]$. Interestingly, we observed significant co-enrichment of multiple histone marks at intergenic regions. The significance of these epigenetic marks at intergenic regions remains elusive. In this study, we identified 462 intergenic enhancer-like elements by analysing their transcriptional-epigenomic status and validated representative enhancers by functional luciferase reporter assays. Thus, our comprehensive analysis of histone modifications identified enhancer-like elements on a genome-wide scale and revealed complex genome architecture that may facilitate spatio-temporal regulation of virulence/pathogenicity genes in Plasmodium falciparum.

\section{Results}

\section{Genome-wide identification of enrichment of histone modifications at intergenic regions}

To gain insights into the epigenomic landscape of $P$. falciparum, we used previously generated chromatin immunoprecipitation sequencing (ChIP-seq) data of multiple histone modifications at three different stages of intra-erythrocytic cycle; rings, trophozoites and schizonts $[18,19]$. Interestingly, we observed enrichment of histone modifications at the intergenic regions (IRs) apart from promoter regions and gene-body. Role of histone modifications is extensively studied on promoters and in gene body, however, little is known about their role at IRs in the Plasmodium genome. Based upon tag density, which defines strength of enrichment of histone modifications (H3K4me3, H3K4me1, H3K9ac, H3K14ac and histone H4ac); we identified 462 IRs (Fig. 1a; Additional file 1: Figure S1 and Additional file 2: Table S4). Interestingly, the profiles of histone modifications have similar pattern (Fig. 1a, left panel) but differential histone modification enrichment (Fig. 1a, right panel). In order to confirm the specificity of the enrichment of histone modifications, we compared publicly available data for H3K4me3 [20] and observed similar pattern (Additional file 1: Figure S2) indicating that both the datasets are of comparable quality and the identified IRs are indeed existent. Furthermore, to confirm identity of the peaks and to rule out the sequence specific biases, we calculated the enrichment of H3K4me3 over pan-H3 on IRs and promoters. Distribution profile and enrichment of H3K4me3 are comparable on these two regulatory elements (Additional file 1: Figure S3), which strengthen our observation that IR peaks are not overrepresented regions of the genome because of sequence biases. As the median intergenic region in P. falciparum is $<2 \mathrm{~kb}$ [21] most of the IRs (80\%) are expectedly located $0.75-2 \mathrm{~kb}$ upstream to the transcription start sites (TSSs) and remaining within $3 \mathrm{~kb}$ distance from the TSSs (Fig. 1b). We also calculated the base-pair (AT/ GC) content at IRs and compared it with the promoters of the protein coding genes in order to see if there are any differences. As expected base-pair composition was found comparable at the IRs and protein coding genes (Fig. 1c). Further to validate the distribution of histone modifications at the IRs obtained in ChIP-seq, we performed ChIP-qPCR at the randomly selected loci for H3K4me1, H3K4me3 and H3K9ac. All the selected histone modifications were enriched at IRs, which were validated by ChIP-qPCR (Fig. 1d).

Transcription regulation is coupled by dynamic events of histone modifications mediated by recruitment and enzymatic activities of histone acetyltransferases (HATs) and histone deacetylases (HDACs) at specific loci in genome. About $30-40 \%$ of histones can be maintained constitutively in low acetylated states without any functional consequence [22]. So, to investigate if histone acetylation marks at IRs are dynamically regulated, we abrogated the enzymatic activity of PfHDACs type I using a HDAC inhibitor, sodium butyrate [23]. Intra-erythrocytic Plasmodium parasites were treated with $10 \mathrm{mM}$ sodium butyrate for indicated time-points and assessed for H3K9ac enrichment relative to total-acetylated $\mathrm{H} 3$ over two randomly chosen intergenic regions (IR1 and IR2) by ChIPqPCR. Within 5 min of HDAC inhibitor (HDACi) treatment, H3K9ac enrichment levels increased by more than 


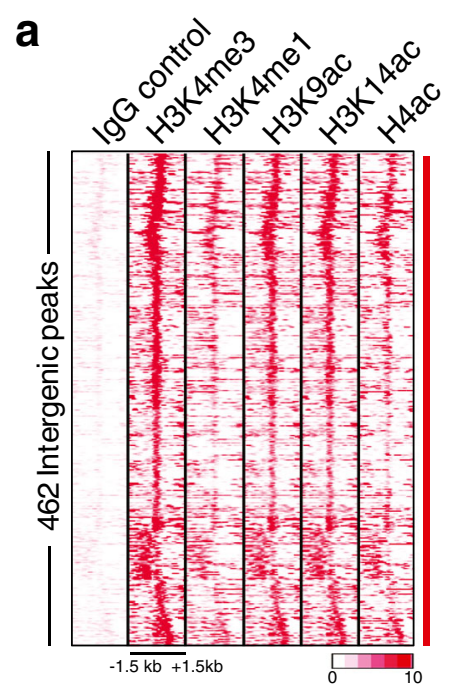

b

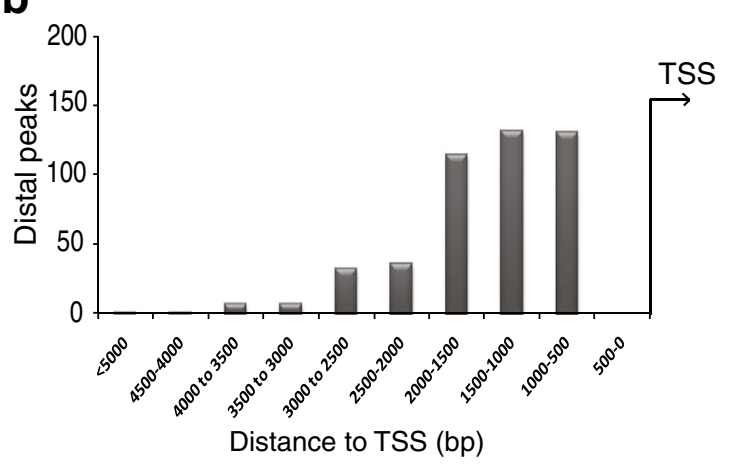

Average Profile

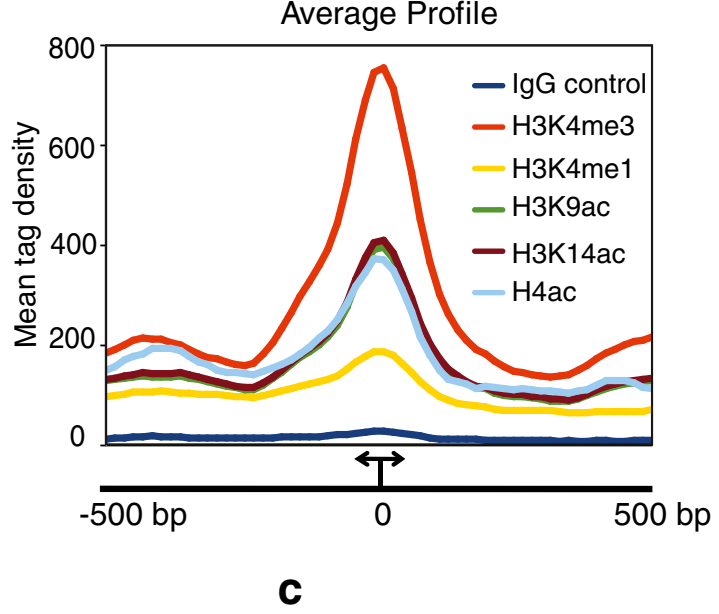

d

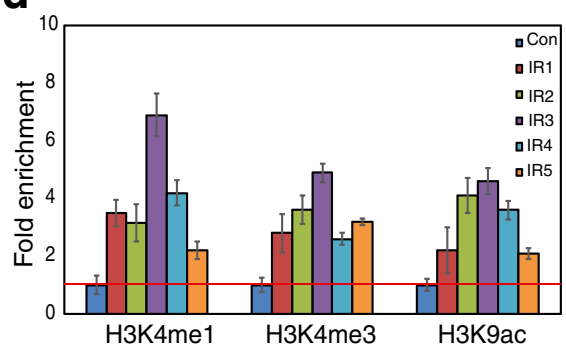

e

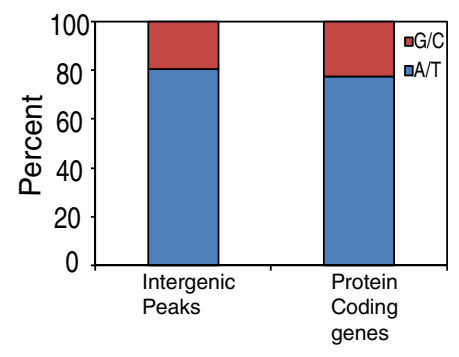

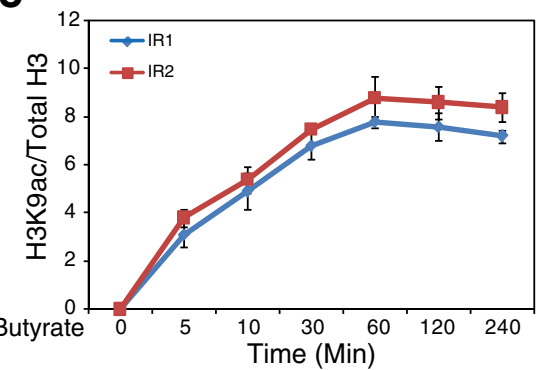

Fig. 1 Identification of intergenic peaks which are associated with multiple histone modifications. In order to extract peaks of histone modifications, tag densities of histone modifications were calculated in the IRs as described in Materials and Methods section. a Heatmap was generated for the region $+/-1.5 \mathrm{~kb}$ around centre of the peak. Tag density of multiple histone modifications over the 462 intergenic peaks (+/$1.5 \mathrm{~kb}$ around centre of the peak) are represented in the heatmap. $\mathbf{b}$ Distribution of intergenic peaks around the transcription start sites was calculated from the centre of the peaks. The closest peak is $750 \mathrm{bp}$ upstream to TSS as the IRs located within 500 bp upstream of the gene have been excluded from the analysis. c AT/GC content of the intergenic peaks and protein coding genes was found comparable as the genome is approximately $80 \%$ AT-rich. d Presence of H3K4me1, H3K4me3 and H3K9ac over the intergenic regions validated by quantitative ChIP-PCR over randomly selected peaks. The ChIP-qPCR enrichment matches with the tag density per peak (data not shown). e Increase in histone H3K9ac over the intergenic peaks following HDAC inhibition by sodium butyrate was measured at indicated time points by ChIP-qPCR. ChIP-signal for histone H3K9ac was normalized to total H3. Control genomic region (primer sequence shown in Additional file 4: Table S1) did not show any increase in acetylation upon sodium butyrate treatment. Error bars represent the standard deviation calculated from three technical replicates 
3-fold, and by 60 min a profound increase of about 8fold was observed (Fig. 1e). This signifies that the histones bound at IRs are certainly dynamically maintained in vivo rather than being constitutively marked by histone modification marks.

\section{H3K4me1 is significantly enriched on intergenic regions} Histone modification marks are predictive of function of the underlying DNA sequences [24]. We wondered if there is any dominant epigenetic mark at IRs as multiple epigenetic marks co-occur at these intergenic regions. We profiled for H3K9ac, H3K4me1, H3K4me3 and Histone H4ac marks over IRs and compared with 500 strong promoters (promoters of genes with high expression) and 500 weak promoters (promoters of genes with low expression). As expected H3K9ac, H3K4me3 and Histone H4ac, which are known gene activation marks, were enriched at the strong promoters (Fig. 2a and b). Interestingly, IRs were significantly $(p<0.05)$ enriched with H3K4me1, a signature mark of enhancer elements (Fig. 2a and b) in higher eukaryotes [25]. H3K36me3, H3K79me3 and H4K20me3 were found comparable at IRs and strong promoters (Additional file 1: Figure S4). Multiple studies have reported H3K4me3 as a hallmark of active promoters and it was also found to be enriched at active enhancers [26]. Hence, classically ratio of H3K4me1 to H3K4me3 is used to distinguish enhancers from promoter elements [27, 28]. We observed significantly higher ratio of H3K4me1 to H3K4me3 occurring at IRs than that observed for strong promoters, indicating that IRs can putatively function as enhancer elements in P. falciparum (Fig. 2c). Surprisingly, weak promoters showed higher ratio of H3K4me1 to H3K4me3 as compared to IRs (Fig. 2c), most probably due to absence of active histone modification (i.e. H3K4me3) over weak promoters (Fig. 2a). Intergenic regulatory elements are found to be enriched for acetylation marks at H3K9 and H3K14 (Figs. 1a and 2a) as reported earlier $[29,30]$. Another hallmark of the regulatory elements is the nucleosome-depleted regions, which can be assessed by Formaldehyde-Assisted Isolation of Regulatory Elements (FAIRE)-sequencing. To assess if the identified IRs, which are also marked by H3K9ac and H3K14ac, can function in vivo as regulatory elements globally; we integrated available FAIRE-seq data in Plasmodium [31] with identified IRs. Interestingly, the identified IRs are depleted of nucleosomes indicating that the predicted IRs are indeed regulatory elements (Fig. 2d).

H3K4me1 enriched intergenic regulatory elements exhibit orientation-independent enhancer reporter activity

To verify the enhancer function of the intergenic regulatory elements, we developed an enhancer luciferase vector by using $\mathrm{pHC1}$ vector as a backbone [32]. Firefly luciferase (FFL) gene was cloned in $\mathrm{pHC1}$ between $5^{\prime}$ PcDT promoter and $3^{\prime}-\mathrm{HRP} 2$ termination signal (Fig. 3a). Randomly selected IR sequences 1 to 7 with putative enhancer properties, negative controls N1 to N3 (randomly selected genomic regions without any histone modifications) were amplified from $P$. falciparum genomic DNA and cloned in pHC1-enhancer luciferase vector upstream of luciferase gene and PcDT 5 ' promoter (Fig. 3a). DNA sequence confirmed clones were used for transient transfection assays in P. falciparum by supplementing parasites with DNA-loaded RBCs [33]. Parasites were cultured for 2 passages and then assayed for luciferase activity as per manufacturer's instructions. The tested IRs (IR 1 to 7) showed enhancer luciferase activity comparable to previously reported enhancer element (H4) of the gene histidine-rich protein 3 (hrp3) from $P$. falciparum (Fig. 3b) [15]. Moreover, luciferase activity was also detected in reverse orientation, whereas luciferase activity in negative controls (N1 to N3), regions lacking enhancer-like properties, was comparable to empty vector control. Exhibition of enhancer luciferase activity by IRs in either orientation suggests that enhancer activity is orientation independent. Thus, the functional enhancer reporter assay demonstrated that IRs may function as enhancer-like elements to regulate transcription of target genes in Plasmodium.

\section{Enhancer-like elements generate non-coding transcripts which positively correlate with the transcription of near- est gene}

Transcription is a characteristic feature of enhancer elements by virtue of RNA Polymerase II occupancy [3436]. Plasmodium enhancer-like elements are also coenriched with H3K4me3, H3K9ac and H4ac (Fig. 2a and b) and H3K36me3, H3K79me3 (Additional file 1: Figure S4) epigenetic marks, which are indicative of active transcription at these elements [18]. So, we mapped RNAsequencing reads to Plasmodium genome to interrogate the transcriptional output from 462 enhancer-like elements. For comparison, we also included RNA-seq reads from 500 strong and weak promoters each, respectively in our analysis. Consistent with observation in higher eukaryotes, transcription of 150-300 bps was observed from enhancer-like elements, while transcription proceeded uni-directionally towards termination site from strong promoters and no transcription was detected from weak promoters (Fig. 4a). Production of noncoding RNAs from IRs is also verified by qRT-PCR over randomly selected loci (Additional file 1: Figure S5).

Recently, a comprehensive map of transcription start sites (TSSs) was reported in Plasmodium falciparum [37]. We observed an overlap of 8 genomic regions between the current set of 462 strong peaks (considering a 


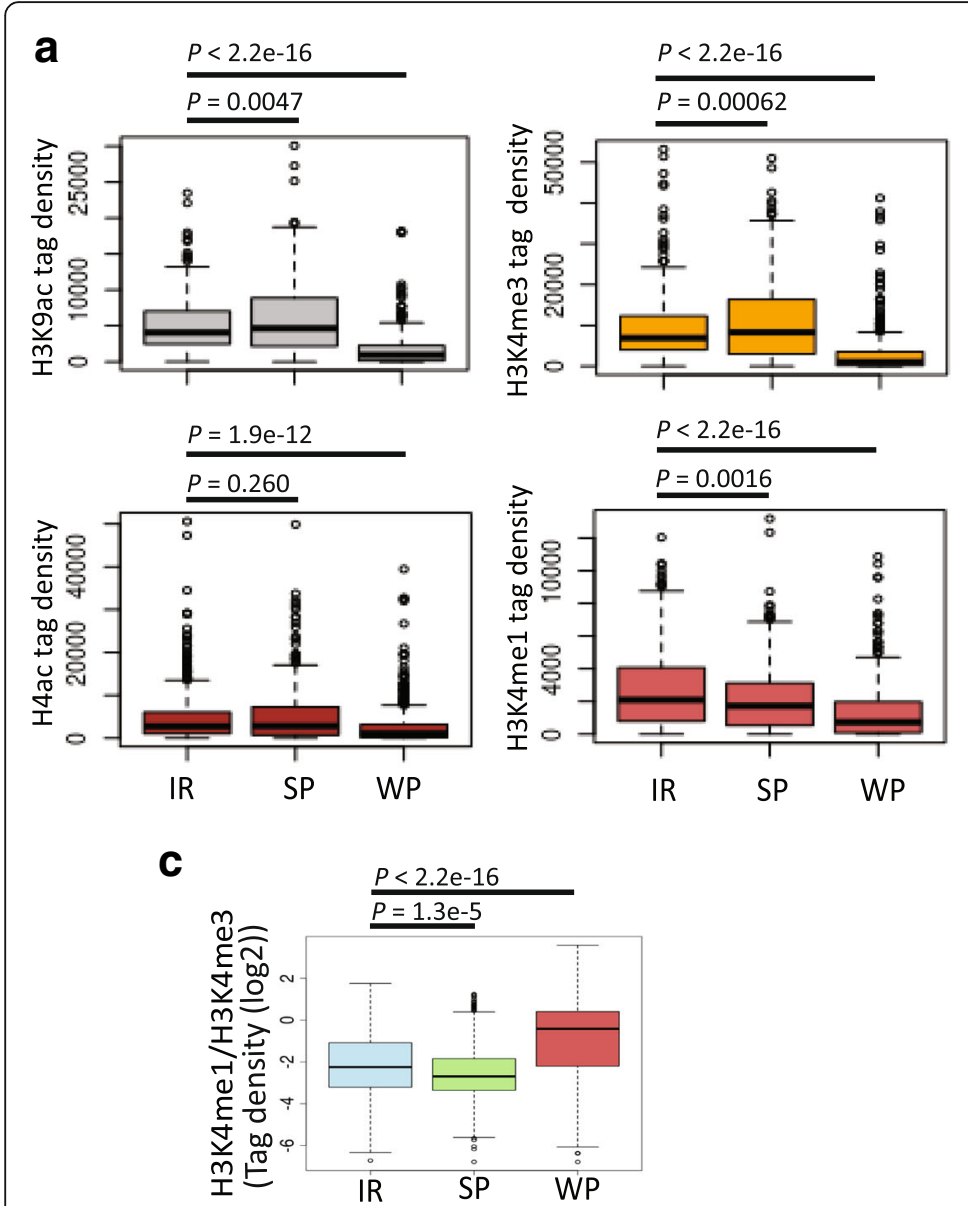

b
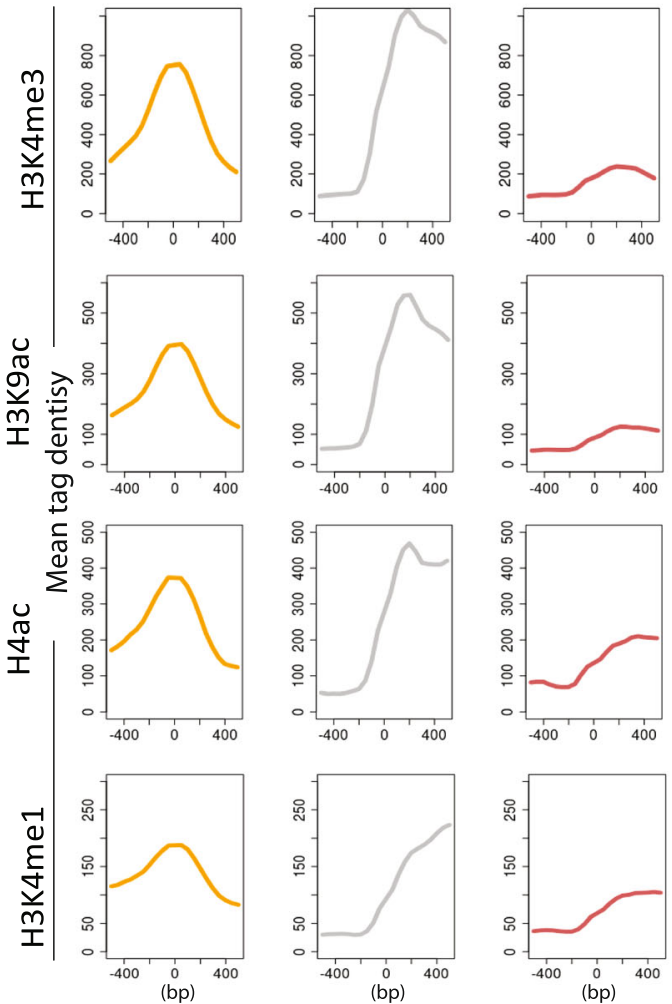

IR peak

TSS-SP

TSS-WP

d

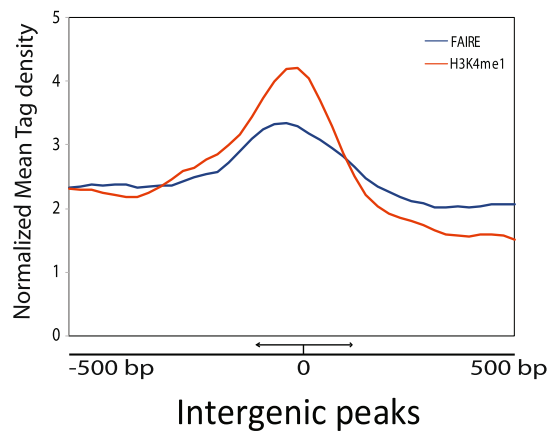

Fig. $2 \mathrm{H} 3 \mathrm{~K} 4 \mathrm{me} 1$ is significantly enriched at the intergenic peaks. a Enrichment of H3K9ac, H3K4me1, H3K4me3 and Histone H4ac over the intergenic peaks and 500 strongly and weakly expressed promoters is calculated. H3K9ac, H3K4me3 and histone H4ac are enriched at the active promoters whereas the distal peaks are enriched with H3K4me1 $(P<0.05)$. IR: Intergenic regions; SP: Strong Promoters; WP: Weak Promoters. $P$ values were computed using the Wilcoxon test (two-sided). b Profiles of H3K9ac, H3K4me 1, H3K4me3 and histone H4ac over the intergenic peaks and 500 strongly and weakly expressed promoters (+/- $0.5 \mathrm{~kb}$ ). Intergenic peaks have bell shaped distribution of all histone modifications. c One of the characteristic features of the enhancer histone modifications in higher eukaryotes, the ratio of H3K4me1 to H3K4me3 tag density is also higher on intergenic peaks as compared to strongly expressed promoters $(P<0.05)$. Weak promoters showed higher ratio of $\mathrm{H} 3 \mathrm{~K} 4 \mathrm{me} 1 \mathrm{to}$ H3K4me3 as compared to IRs, most probably due to absence of active histone modification (i.e. H3K4me3) on weak promoters d. Furthermore, one of the hallmarks of the regulatory elements is the nucleosome-depleted regions, which can be assessed by Formaldehyde-Assisted Isolation of Regulatory Elements (FAIRE)-sequencing. To assess if the identified IRs can function in vivo as regulatory elements globally; we integrated available FAIRE-seq data in Plasmodium with enrichment of H3K4me1 on identified IRs. Overlapping peaks of H3K4me1 and FAIRE-sequencing over the 462 intergenic peaks suggests that identified IRs are depleted of nucleosomes and that the intergenic peaks are indeed regulatory elements 

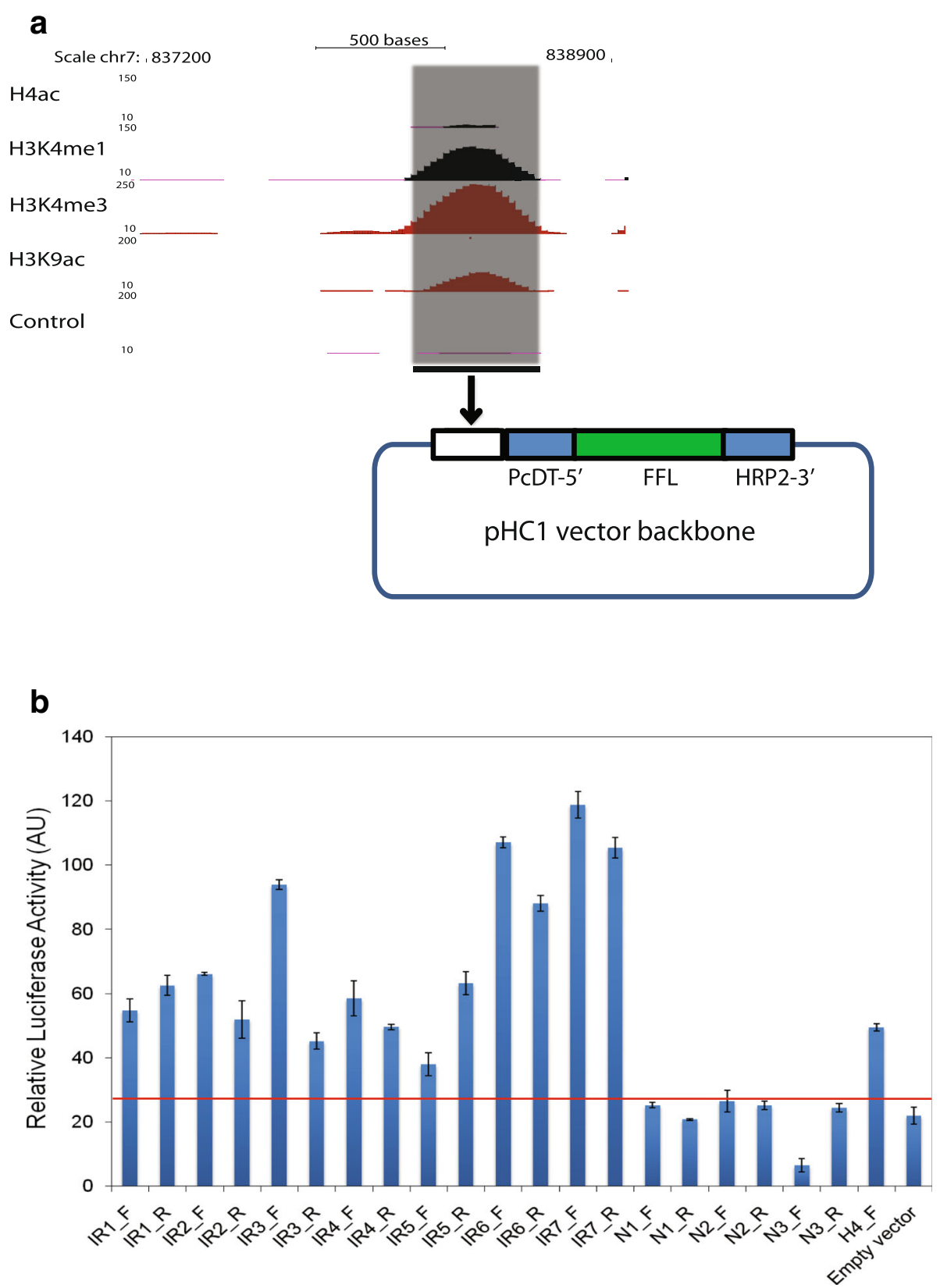

Fig. $3 \mathrm{H} 3 \mathrm{~K} 4 \mathrm{me} 1$ marked intergenic peaks exhibit enhancer activity in enhancer luciferase assay. a UCSC snapshot of a representative intergenic peak having multiple histone modifications. The intergenic region $(\sim 1 \mathrm{~kb})$ was cloned in an enhancer luciferase reporter vector. $\mathbf{b}$ Enhancer reporter (luciferase) assay was carried out to measure the activity of seven randomly selected intergenic regions (IR1 to IR 7) and compared to randomly selected negative genomic regions (N1-N3) and empty vector control in P. falciparum. F denotes forward orientation, whereas its opposite orientation (Reverse) is denoted as R. Earlier reported enhancer (H4) [15] was used as positive control. Intergenic regions showed enhancer activity in the enhancer luciferase assay independent of orientation. The red line indicates the activity obtained with control negative genomic region. Error bars represent the standard deviation for two biological replicates

region of $250 \mathrm{bp}$ up/down of a peak centre) and 1580 newly reported TSSs (Additional file 3: Table S5). Six of these overlapping enhancer-like elements are smaller than 115 nucleotides indicating that ORFs produced from them are very small $(<30$ amino acids), which in turn suggest that they might be acting as enhancer-like elements rather than promoter. Nevertheless, nonoverlap between 454 intergenic peaks reiterates that the regions we have identified are not unannotated promoters. We wondered if the RNA produced from enhancer-like elements has protein coding potential and the identified intergenic regions are in-fact unannotated 

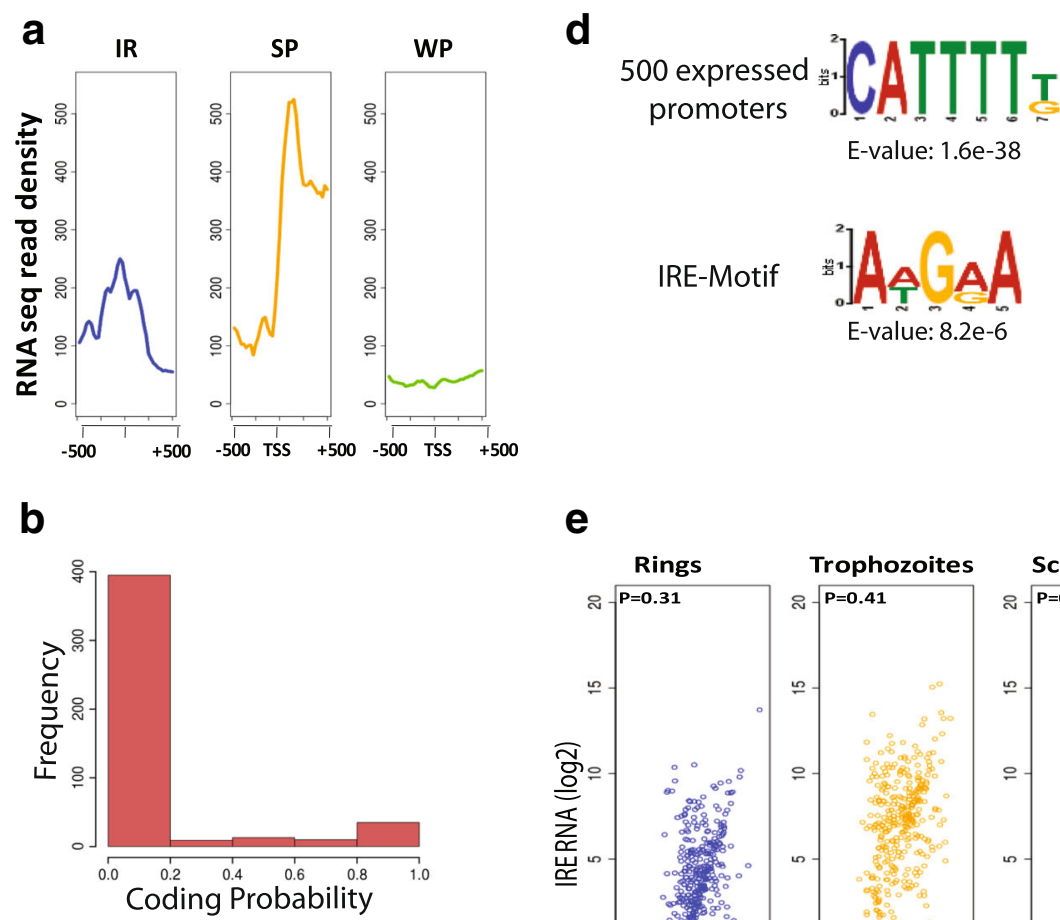

e
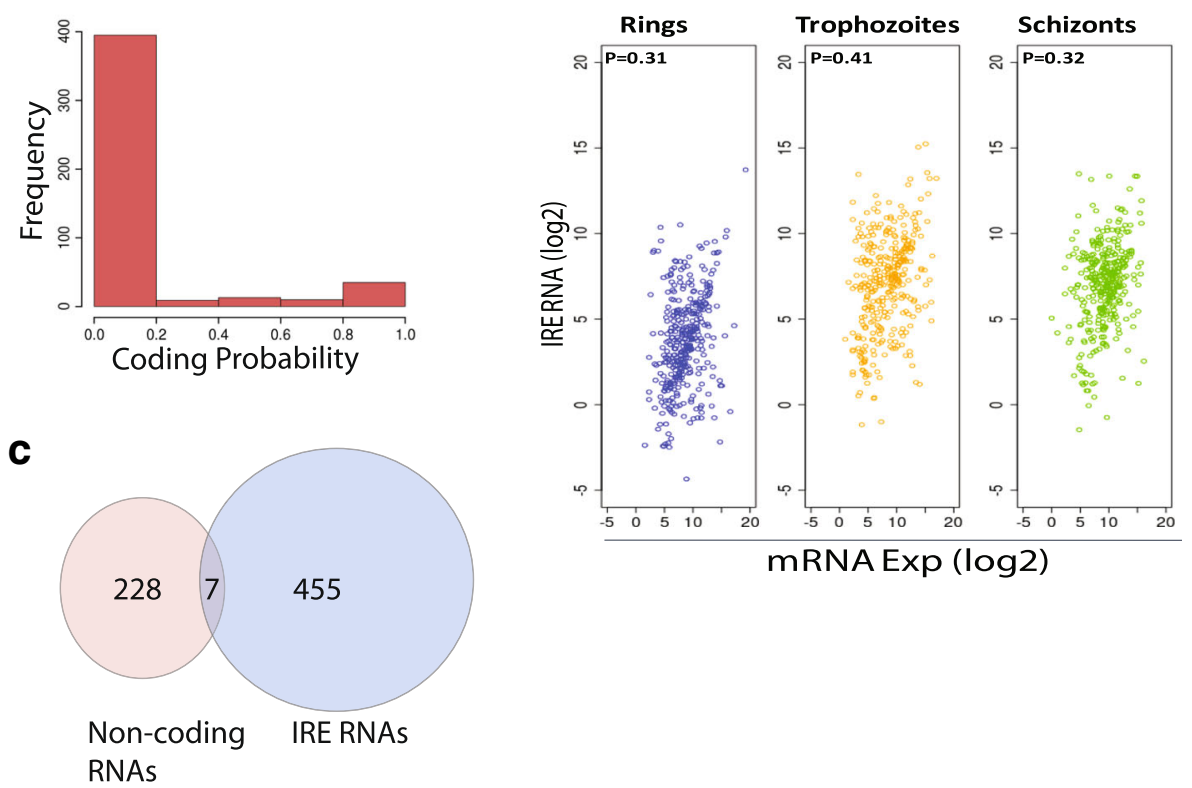

Fig. 4 Enhancer-like intergenic peaks produce short RNAs which positively correlates with the expression of nearest gene. a Distribution of RNAsequencing reads is plotted over the intergenic peaks and 500 strongly and weakly expressed promoters. Average distribution of RNA-sequencing reads suggests that intergenic peaks produce short (150-300 bps) bi-directional transcripts. b Most of the enhancer-like element RNAs do not exhibit protein coding potential. c Only 7 of the enhancer-like element RNAs overlap with known non-coding RNAs suggesting that enhancer-like element RNAs are novel non-coding RNAs. $\mathbf{d}$ The consensus motif at the enhancer-like element is different than the motif found at the highly expressed promoters indicating involvement of different set of transcription factors. e Correlation in the expression of enhancer-like element RNAs from the intergenic peaks is calculated with the expression of nearest gene in a stage specific manner. Enhancer-like element RNAs produced from intergenic regulatory peaks showed moderately positive correlation with the expression of nearest neighbourhood gene

promoters. We first tested the accuracy of the known tools for predicting coding potential of a given nucleotide sequence from P. falciparum. A total of 5536 non-redundant protein coding and 1563 non-coding RNA sequences (snRNA, tRNA, rRNA, snoRNA and miscellaneous RNA from all Plasmodium species) were extracted from PlasmoDB (http://plasmodb.org). Coding Potential Calculator (CPC) was found to predict 100\% known non-coding RNAs from Plasmodium (Additional file 1: Figure S6). Coding potential of enhancer-like elements by CPC suggest that more than $90 \%$ of the identified intergenic elements have no protein coding potential (Fig. 4b). Interestingly, only 7 of the 462 enhancer-like element RNAs overlap with known non-coding RNAs (Fig. 4c). Furthermore, the most frequently occurring motif enriched at enhancer-like element sequences is significantly different than the motifs enriched at the promoters of highly expressing genes (Fig. 4d) and randomly selected intergenic regions (Additional file 1: Figure S7). TomTom search identified that the enhancer-like element motif - AWGRA has similar recognition sequence as found for some of the ApiAp2 TFs namely PF11_0404_D1, PFL1900w_D1, and PF13_0267; but as indicated the E-values are higher reducing the confidence-level (Additional file 1: Figure S8). 
Collectively, these indicate that the enhancer-like element RNAs are novel non-coding RNAs.

In higher eukaryotic systems enhancers are shown to regulate the transcription of nearest neighbourhood gene and their RNA (eRNA) level correlates with expression of the nearest gene [35]. We wondered if it is also true for enhancer-like element RNAs and so we calculated the correlation in expression between enhancer-like element RNAs and nearest neighbourhood gene expression. The expression of transcribed enhancer-like element RNAs correlates positively with the expression of nearest neighbouring genes at ring, trophozoite and schizont stages of IEC as evident from positive Pearson correlation coefficient values $0.31,0.41$, and 0.32 , respectively (Fig. 4e). The moderately positive Pearson correlation coefficients between different stages indicate presence of additional layers of contacts playing a role in stage specific transcriptional regulation. This in turn suggests that enhancer-like element RNAs could have a functional role in regulating transcription.

\section{Enhancer-like elements are clustered spatially over virulence-pathogenicity genes}

Eukaryotic genomes are organised spatially into higher order structure to accommodate chromatin in smaller nuclear space. The three-dimensional genome architecture is defined by its physical division into topological domains within which chromatin looping mechanisms mediate enhancer-promoter interactions [38]. In order to investigate the topological contacts of enhancer-like elements globally, we mapped $\mathrm{Hi}-\mathrm{C}$ data generated at $10 \mathrm{~kb}$ resolution of the spatial organization of $P$. falciparum genome at three different stages [39]. Enhancerlike elements are involved in intra-chromosomal (contacts formed within same chromosome i.e. cisinteractions) as well as inter-chromosomal (contacts formed with regions from another chromosome i.e. trans-interactions) interactions with gene promoters. Cis-interactions of enhancer-like elements with gene promoters are more ubiquitous than trans-interactions (Fig. 5a). We filtered the Hi-C data to get contact information of the 462 enhancer-like elements with the genes falling within $1 \mathrm{~kb}$ of the target promoter regions. The total number of such genes is 959. Overall, number of contacts increased from rings (1203) to trophozoites (2247) to schizonts (9732) indicating either tighter mechanical packaging of chromatin or stage-specific dynamic role of enhancer-gene contacts. Furthermore, we have taken equal number (462) of random non-enhancer like genomic sequences and performed the analysis for contact information. As the resolution of the $\mathrm{Hi}-\mathrm{C}$ data is $10 \mathrm{~kb}$ and chromatin packaging varies across the different stages, we observed linear increase in contacts from the rings to trophozoites to schizonts indicating tighter mechanical packaging of chromatin during parasite growth. However, random non-enhancer like sequences do not show higher contacts per gene suggesting that higher number of contacts formed by enhancer-like sequences are specific (Additional file 1: Figure S9). Moreover, on an average, an active enhancerlike element contacts 9.5 promoters while a promoter contacts 4.5 enhancers, suggesting enhancer-like elements are clustered spatially with multiple genes in nucleus (Fig. 5a). More number of contacts has been established for the virulence genes than other genes in Plasmodium genome [39, 40], additionally, our study showed that genes with more than 5 contacts are involved in Plasmodium pathogenesis like cytoadherence and rosetting while genes with 5 or less contacts are involved in housekeeping functions (Fig. 5b). The biological implications for segregation of genes into housekeeping and virulence-pathogenicity clusters point towards the existence of differential transcriptional control. Clustering of virulence genes has been shown by DNA fluorescence in situ hybridization (FISH) in previous studies [41, 42]. Here, we hypothesize that clusters with more number of interactions among enhancer-like elements and genes fine-tune the transcriptional program for co-regulated control over virulencepathogenicity gene expression. Though further experimentations are required, the observations highlight involvement of genomic context i.e. nuclear architecture, micro-environment within transcriptional clusters like availability of some nuclear factors (e.g. transcription factors, chromatin remodellers) in enhancer functionality.

\section{Discussion}

Identification of enhancer elements in $P$. falciparum has been challenging largely due to their property to work in location-, orientation- independent manner; additionally AT-richness of Plasmodium genome makes it difficult to identify regulatory elements [43]. Furthermore, functional validation of identified regulatory element is difficult as manipulation of $P$. falciparum genome is hard to achieve. In this study, we have integrated ChIP-seq data sets for multiple histone modification marks to identify and characterize novel enhancer-like elements with their potential role in transcription regulation in $P$. falciparum.

\section{Intergenic sequences are enhancer-like elements}

We illustrate that the identified intergenic regions exhibit features similar to those observed at transcriptional enhancer elements in higher eukaryotic systems. The assurance about identified enhancer-like elements not being unannotated promoters is supported by multiple observations as stated herein. First, a distinctive 


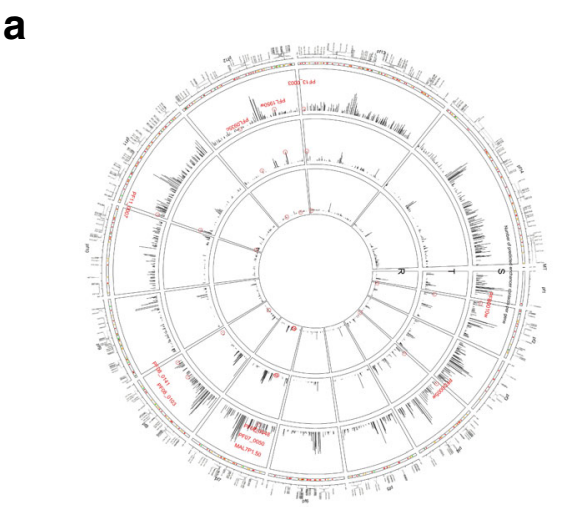

b

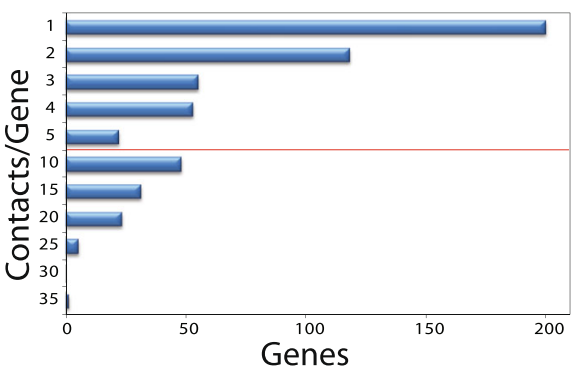

Genes with more than 5 contacts/gene ( $p$-value <0.005)

Cytoadherence to microvasculature
Rosetting
Cell-cell adhesion
Pathogenesis
Cell communication
Antigenic variation

Fig. 5 Enhancer-like intergenic peaks form multiple contacts in the genome and exhibit stage specific functions. a Circular plot represents number of contacts made by the $P$. falciparum genes with enhancer-like elements and their correlation with gene expression. Overall, enhancer-gene body contacts increase from rings (R) to trophozoites (T) to schizonts (S) stage. The inner three tracks labelled with $S, T$ and R show bar plots for the number of contacts per gene in the particular stage. There are total 959 genes (includes 11 virulence genes) contacting enhancers with maximum number of contact made by a gene is 58 . Out of 959 genes, 366 genes have strong positive or negative correlation between the number of contacts and gene-expression. These are shown as colored dots along with the gene label on each chromosome from pf1 to pf14 and MT (mitochondrial) in the outermost two tracks. The color codes for the dots are: Dark green represents correlation value ranging from -1.0 to -0.9 ; Green represents correlation value ranging from $>-0.9$ to -0.7 ; Orange represents correlation value ranging from 0.7 to $<0.9$; Red represents correlation value ranging from $>0.9$ to 1.0 . Interestingly, the virulence genes (represented by red circles in each track S, T and R) show highest enhancer contacts in T stage. These virulence genes are labelled in red texts. 6 out of 11 virulence genes (PFB0010w, MAL7P1.50, PF08_0141, PF08_0103, PFL0935C and PF13_0003) show strong positive correlation (> = 0.7) between the contacts made by them and their expression. The correlation between number of contacts of genes with enhancers and its expression across three stages is calculated using Pearson method. b Number of contacts established per gene by enhancer-like elements is calculated for trophozoite stage. Gene ontology of genes showing more than 5 contacts and equal to or less than 5 contacts per gene. Genes with more than 5 contacts are mostly involved in the complex regulation pathways however; genes showing equal to or less than 5 contacts are involved in the maintenance of housekeeping function

epigenetic profile, H3K4me1 as the significantly enriched histone modification mark and higher H3K4me1/ H3K4me3 ratio persists at enhancer-like elements in comparison to promoters [27, 28]. Second, the intergenic regions displayed orientation-independent enhancer activity in transient firefly luciferase assay confirming their functionality as enhancer elements in P. falciparum. Third, characteristic bi-directional transcription is observed from enhancer-like elements $[44,45]$ while transcription proceeds uni-directionally from promoter elements (Fig. 4a). Furthermore, the transcripts generated from enhancer-like elements are non-coding RNAs (Fig. 4b and c). And fourth, distinct motif is enriched in enhancer-like elements than at promoters (Fig. 4d). In higher eukaryotic systems, classical histone marks of enhancers are H3K4me1, H3K4me3, H3K27ac and H3K27me3 [46, 47]. Acetylation and methylation of H3K27 permit to distinguish between active and inactive/poised enhancers, respectively. Interestingly, $P$. falciparum lacks H3K27me3 $[18,48]$ and seems to have 
very low levels of H3K27 acetylation [18], suggesting that identified intergenic regions are enhancer-like elements with a potential distinct signature in $P$. falciparum.

\section{Intergenic regulatory element RNAs- a novel class of non- coding RNAs which positively correlates with expression of nearest neighbourhood genes}

The chromatin state at enhancer-like elements appears to be transcriptionally active owing to nucleosome-depleted region [18, 31]. Comprehensive epigenomic mapping of regulatory elements revealed that multiple histone modification marks persist at enhancers in higher eukaryotic systems [49-51]. Similarly, the genomic loci of enhancer-like elements are enriched with multiple activations marks (eg. H3K4me3, H3K9ac etc) in P. falciparum [18, 41]. Thus, enhancer-like elements also gain transcriptionally active states equivalent to that of coding regions and produces RNAs. These enhancer-like element RNAs, which are equivalent to enhancer RNAs (eRNAs) in higher eukaryotic system, are a new class of non-coding RNA in Plasmodium that are transcribed bi-directionally from enhancer-like elements. Interestingly, bi-directionally transcribing enhancer RNAs have also been used to predict active enhancers [34, 52]. Experiments from mammalian cell lines implicate eRNAs in a variety of functions such as chromatin looping, recruiting histone acetyltransferases, augmenting chromatin accessibility, depositing H3K4me1/2 marks at enhancers, and increasing recruitment of RNA Polymerase II at gene promoters $[43,44]$. As of now, we have no interpretation for the biological functionality of enhancer-like element RNAs, but their expression positively correlates with the expression of the nearest neighbouring gene along IEC of $P$. falciparum implying that a substantial proportion of enhancer-like elements can harbour their target genes in immediate vicinity and can act by mechanisms involving enhancer-like element RNAs. Deletion of the enhancer-like elements in the genomic context should provide final evidence of functional activity. Unfortunately, generating transgenic lines in P. falciparum is non-trivial, limiting the use of modern techniques in deciphering the role of enhancer-like elements. Since, most of the genes are poised in P. falciparum [18], further systematic investigation of the function of enhancer-like element RNAs should reveal the diverse transcriptional regulatory mechanisms that orchestrate $P$. falciparum's life cycle and may identify novel mechanisms that can be targeted for future drug-development against malaria parasite.

\section{Intergenic regulatory elements, spatial genome organization and regulation of clonally variant multicopy genes}

Epigenetic profile at enhancer-like elements does not change drastically along the developmental stages of parasite suggesting an additional layer of transcriptional regulation in $P$. falciparum, which is different than stimulus-dependent enhancer activation in higher eukaryotic systems [53, 54]. We postulate that the dynamic global genome re-organisation events during IEC participate in spatially organising the enhancer-like elements that lead to establishment of their interactions with target gene promoters [31, 39]. In genome-wide nuclear architecture context, enhancer-like elements are involved predominantly in intra-chromosomal than inter-chromosomal interactions with genes. This observation is consistent with earlier report that majority of DNA interactions are contained within intra-chromosomal structures $[39,55]$. Enhancer-like elements are involved in spatial clustering with multiple genes in threedimensional nuclear architecture. Plasmodium genome is known to be partitioned into active and repressed zones for transcriptional regulation of virulence genes [56] and active transcription occurs at few discrete locations that appear to be developmentally regulated along the IEC of Plasmodium [42]. Numerous studies suggest that genomic loci of virulence-pathogenicity genes, which are clonally variant multicopy (CVM) genes, possess characteristically distinct epigenetic features. CVM genes are particularly marked by histone $\mathrm{H} 4$ acetylation activation mark and H3K9me3 repression mark [18, 41]. Involvement of this combinatorial pattern of epigenetic marks in segregating genes into housekeeping and virulence-pathogenicity clusters needs further investigation. Identification of molecular players involved in segregating and maintaining functionally linked genes as clusters would enable genetic manipulations to disrupt clusters of enhancer-like elements and virulence-pathogenicity genes. Genes with more than 5 contacts are all involved in mechanisms of pathogenesis like rosetting, cytoadherence and virulence; while those with 5 or less contacts are associated with genes of housekeeping functions. The biological implications for segregation of genes into housekeeping and virulence-pathogenicity clusters point towards the existence of differential transcriptional control. Clustering of virulence genes (2 to 5 clusters per cell) have been shown by DNA fluorescence in situ hybridization (FISH) in previous studies $[39,55]$. Here, we hypothesize that clusters with more number of interactions among enhancer-like elements and genes fine-tune the transcriptional program for co-regulated control over virulencepathogenicity gene expression. Though further experimentations are required, the observations highlight involvement of genomic context i.e. nuclear architecture, micro-environment within transcriptional clusters like 
availability of some nuclear factors (e.g. transcription factors, chromatin remodellers) in enhancer functionality.

Plasmodium codes for Apicomplexan Apetala2 (ApiAp2) family of proteins and are the major known transcription factors in genome [10]. Genome-wide DNA-binding motifs have been validated for ApiAp2 transcription factors and members of ApiAp2 family are known to bind numerous distinct motifs apart from the highly preferred primary motif. The complexity increases further as ApiAp2 TFs can form homo- and heterodimers [57-60]. The enhancer-like element motif AWGRA seems to be a sequence extension of ApiAp2 core motifs namely PF11_0404_D1, PFL1900w_D1, and PF13_0267 plausibly suggesting their involvement in transcriptional control at enhancer-like elements. The transcriptional co-activator PfGCN5 along with its acetylated target $\mathrm{H} 3 \mathrm{~K} 9 / \mathrm{K} 14$ has been reported to be enriched at a previously known pf1-cys-prx enhancer element $[16,61]$. Our data demonstrates that the nucleosomes positioned at identified enhancer-like elements are marked by H3K9 and H3K14 acetylation, implicating PfGCN5 as a transcriptional co-activator at enhancerlike elements. Assembly of protein complex at enhancers called the enhanceosome complex requires acetylation by GCN5, which eventually leads to recruitment of transcription machinery [62]. Future research on Plasmodium enhanceosome will aid in our understanding of the concerted action of sequence- and/or structure-specific transcription factors, chromatin modifiers and remodelers that participate in transcriptional regulation mediated by enhancer-like elements.

\section{Conclusions}

In conclusion, this study identified genome-wide intergenic regulatory elements in P. falciparum that function as transcriptional enhancers. As observed in higher eukaryotes such as human and mouse, intergenic regulatory elements produce non-coding RNAs, which positively correlate with the gene expression. Even though the function of these elements remains to be established individually in $P$. falciparum, our study is the first report in any lower eukaryotic parasitic system where the enhancers are characterized in such greater details. Thus, this report contributes to the fundamental understanding of parasite regulatory genomics by first time identification of novel genome-wide enhancerlike elements, defining their epigenetic and transcriptional features and provides a resource of functional cisregulatory elements that may give insights into the virulence/pathogenicity of Plasmodium falciparum.

\section{Methods}

\section{Culturing of Plasmodium falciparum parasites}

P. falciparum culture-adapted parasite lines were kindly provided by Dr. Vasudevan Seshadri (National Center for Cell Science, Pune) and were cultured as previously described [63]. Briefly, parasites were cultured at 10\% parasitemia in RPMI1640 medium (Pan-Biotech, Germany) supplemented with $25 \mathrm{mM}$ HEPES, 0.5\% AlbuMAX I, $1.77 \mathrm{mM}$ sodium bicarbonate, $100 \mu \mathrm{M}$ hypoxanthine and $12.5 \mu \mathrm{g} \mathrm{ml}^{-1}$ gentamicin sulphate at $37{ }^{\circ} \mathrm{C}$. Parasites were subcultured by replacing the entire spent medium and splitting the culture into two or more flasks to maintain parasitemia to $10 \%$ and quickly restoring the hematocrit to $1 \%$ in the required volume of culture medium.

\section{Data source and analysis}

ChIP-seq datasets were downloaded from the public data bank Gene Expression Omnibus (http://www.ncbi.nlm.nih.gov/gds) under the accession number GSE63369 (H3K4me3, H3K4me1, H3K9ac, H3K14ac, H4ac, PanH3). ChIP-sequencing data for histone modifications (H3K36me2, H3K36me3 and H4K20me3) and RNAsequencing (GSE23865) were downloaded from the public databank Gene Expression Omnibus for P. falciparum $[64,65]$. Chromosome conformation capture coupled with next-generation sequencing technology $(\mathrm{Hi}-\mathrm{C})$ contact data were used from earlier study [39]. GSE50199 (GSM1215592, GSM1215593 and GSM1215594) for coordinates for contacts from $\mathrm{Hi}-\mathrm{C}$ at ring, trophozoite and schizont stages, respectively. Formaldehyde-assisted isolation of regulatory elements to extract protein-free DNA (FAIRE) data (SRR030738) was integrated from earlier study [31]. The scatter plots, k-means clustering and average gene profiles were created using seqMINER $[31,66]$. Box plots and correlation analysis were produced using ' $R$ ' software (http://r-project.org/). Sequences of primers used for quantitative ChIP-PCR validation are listed in Additional file 4: Table S1.

\section{Establishment of list of intergenic regions enriched for histone modifications}

Transcription start sites (TSSs) and transcription termination sites (TTSs) of coding gene in P. falciparum were determined as described earlier [18]. Intergenic regions were selected by excluding gene unit (from TSS to TTS as described earlier [18] and $+/-500$ bp around gene unit. A total of 462 histone modification peaks were identified in the entire $P$. falciparum intergenic genome using seqMINER [66].

\section{Visualization of histone modifications on intergenic regions}

We extracted the tag density in a $1.5 \mathrm{~kb}$ window surrounding the intergenic peaks using the program seqMINER which generates heatmap as well as the profiles [66]. The sequenced ChIP-seq reads represent only the end of each immunoprecipitated fragments instead of 
the precise protein-DNA binding sites. To illustrate the entire DNA fragment, essentially before analysis, 3 ' end of each ChIP-seq read was extended to $200 \mathrm{bp}$ in the direction of the reads. Data is normalized by dividing the reads per bin by total reads per modification.

\section{Distance plot calculation}

Distance from Transcription Start Sites (TSSs) for each intergenic peak was calculated from the centre of the intergenic peak to the nearest TSS. The distribution of peaks is plotted for every 500 bps around the TSSs.

\section{Calculation of average length of intergenic peaks}

P. falciparum3D7_Genome_v9.3 was used to calculate the length of all the genes in P. falciparum. Length of intergenic peaks is calculated by taking the mean tag density over the intergenic peaks in a window of $+/-$ 1000 bps. Background tag density was used to put an arbitrary cut-off for determining the average intergenic peak length.

\section{Calculation of distribution of histone modifications marks over intergenic peaks and promoters}

Total tag density of histone modifications was calculated as described above in average profile calculation over the strong intergenic peaks, 500 strongly and weakly expressed promoters. The profiles of histone modifications over the intergenic peaks, strongly and weakly expressed promoters are calculated using seqMINER [66].

\section{Construction of luciferase vector system to test the enhancer activity of intergenic peaks}

$\mathrm{pHC1}$ vector (obtained from BEI resources) was used as a template to develop a enhancer luciferase system for Plasmodium falciparum. pHC1 vector was first digested with the HindIII restriction enzyme to remove the extra set of promoter and 3' UTR (CAM5' promoter and HSP86-3' UTR) and re-ligated using T4 DNA ligase. Later Tg-DHFR-TS gene was replaced with the Luciferase gene from pGL3 promoter vector (Promega) and resultant vector is called $\mathrm{pHC1}$ enhancer vector. Predicted intergenic sites enriched with H3K4me1 and negative regions devoid of any regulatory marks $(\sim 1 \mathrm{~kb})$ were amplified from Plasmodium genomic DNA and cloned into pHC1 enhancer vector in single HindIII restriction site upstream of luciferase gene and $\mathrm{PcDT} 5^{\prime}$ promoter. Confirmation of orientation was performed by restriction mapping and DNA sequencing. Co-ordinates of the cloned sites and primer information are provided in Additional file 1: Table S2.

\section{Enhancer luciferase reporter assay}

Equal amount of parasites were cultured on RBCs loaded with indicated DNA constructs for 2 passages. Culture was spun at $1500 \mathrm{rpm}$ for $5 \mathrm{~min}$, and the pellet subjected to saponin lysis in $1 \mathrm{X}$ PBS for $10 \mathrm{~min}$ at $4^{0} \mathrm{C}$. Parasite pellets obtained by centrifugation at $2800 \mathrm{rpm}$ for 5 min were washed with $1 \mathrm{X}$ PBS to remove haemoglobin. Parasites were lysed in $30 \mathrm{uL}$ of $1 \mathrm{X}$ Passive Lysis Buffer by incubating on ice for $10 \mathrm{~min}$. Luciferase assay was performed with Promega Kit as per instructions. Lysates were spun at top speed for $3 \mathrm{~min}$ and $20 \mathrm{uL}$ of supernatant is added in $100 \mathrm{uL}$ of Luciferase Assay Reagent II (LAR II) and firefly luciferase activity measured luminometrically. Luminescence Units were expressed as fold change above the empty vector control and also normalised with parasitemia counts measured at the time of harvesting. Experiments were performed at least twice.

Processing of RNA-seq reads to visualize the distribution over the intergenic peaks and promoters and its correlation with expression of nearest neighbourhood gene Genes were categorized based on increasing expression levels (based on reads assigned per kilobase of target per million mapped reads (RPKM)) using the RNA-seq data (GSE23865). Datasets for different stages were normalized for the total number of uniquely mapped tags. Stage specific RNA-Seq data was corrected for each stage by a correction factor (based on the amount of RNA per parasite nucleus) present in each stage as described earlier. Distribution of RNA-seq reads over the intergenic peaks, and 500 strongly and weakly expressed promoter is calculated using the seqMINER [66]. Correlation between the RNA produced from the intergenic peaks is calculated with the expression of nearest neighbouring gene (closest distance from transcription start site irrespective of upstream or downstream of the gene unit).

\section{Circular plot}

Circular plot was generated in $\mathrm{R}$ v3.2.2 using circlize v0.3.5 package of CRAN. The genomic interactions observed in Hi-C data [39] were filtered at FDR $<0.01$. The filtered dataset has 7830 interactions in ring stage (Additional file 5: Table S3 Rings_contact_FDR0.01), 18,320 interactions in trophozoite stage (Additional file 5: Table S3 Trophozoites_contact_FDR0.01) and 65,534 interactions in schizont stage (Additional file 5: Table S3 Schizonts_contact_FDR0.01). The contact information is extracted as described: let us assume a scenario where a genomic locus GL1 interacts with another genomic locus GL2 (both from Hi-C data), a Peak P1 (from ChIP-seq data) overlaps with GL1, and a Gene Y is within $1 \mathrm{~kb}$ to GL2. Thus, it indicates that Peak P1 is interacting with the Gene Y. Likewise, we determined all 
the overlapping genomic loci GL1 for each peak P1 considering there is at least 50 bp overlap between $250 \mathrm{bp}$ up- and down-stream of the peak centre and $5 \mathrm{~kb}$ upand down-stream of the genomic locus. We considered only those Peak (P1)-Gene(Y) interactions where distance between Gene Y and GL2 is $<1 \mathrm{~kb}$. There are 1203 such interactions in Ring stage, 2247 interactions in Trophozoite stage and 9732 interactions in Schizont stage (Additional file 5: Table S3 Rings_1kb_contacts, Trophozoites_1kb_contacts and Schizonts_1kb_contacts). For each gene $\mathrm{Y}$, all the enhancer contacts (P1-Y interactions) are counted (Additional file 5: Table S3 Rings_Enhancer_contacts_perGene, Trophozoites_Enhancer_contacts_per Gene and Schizonts_Enhancer_contacts_per Gene). We have used RNA-Seq data (GSE23865) to get the gene-expression values for the genes (Additional file 5: Table S3 Exp_RTS). The correlation between enhancer contacts and gene-expression values for each gene is calculated in $\mathrm{R}$ using Pearson method (Additional file 5: Table S3 Count_Exp_cor). For this analysis, we have used PlasmoDB-9.3_P. falciparum 3D7_Genome (http://plasmodb.org/common/downloads/ release-9.3/Pfalciparum3D7/).

\section{Calculation of AT/GC content}

Nucleotide sequence of the 462 enhancer-like elements were prepared by fetching 250 bases up and down of the peak centre using P. falciparum3D7_Genome_v9.3, and per base GC content and per sequence GC content was calculated. The average GC\% in 5398 protein coding genes (downloaded from www.plasmodb.org) and the 462 enhancer sequences were 22.867 and 20.949, respectively. GC\% of a sequence was calculated by dividing the number of $\mathrm{G}$ or $\mathrm{C}$ nucleotides in the sequence by the sequence length, and multiplying with 100. Similarly, the $\mathrm{GC} \%$ at a base position in the enhancer sequences was calculated by dividing the number of $\mathrm{G}$ or $\mathrm{C}$ nucleotides at the position by the number of enhancer sequences accounted for that position, and multiplying with 100 .

\section{Motif analysis}

Consensus sequence was determined using MEME-ChIP (meme-suite.org/tools/meme-chip). It is a comprehensive motif analysis tool for large nucleotide sequences. Input DNA was MALARIA (Plasmodium falciparum) DNA and database was Malaria (Campbell et al. [59]). The program was run using default parameters.

\section{Prediction of coding potential of the sequences}

Tools tested for predicting coding potential of given sequence are CPC (Coding Potential Calculator; http://cpc.cbi.pku.edu.cn/), PLEK (Predictor of Long noncoding RNAs and messenger RNAs based on an improved k-mer scheme; http://sourceforge.net/projects/plek/), CNCI (Coding-Non-Coding Index; https:/github.com/ www-bioinfo-org/CNCI) and CPAT (Coding Potential Assessment Tool; http://lilab.research.bcm.edu/cpat/ index.php).

\section{Chromatin immunoprecipation (ChIP) and ChIP-qPCR}

Chromatin immunoprecipitation (ChIP) and ChIP-qPCR are performed as described previously $[18,67]$. Infected RBCs were cross-linked with $1 \%$ formaldehyde (Catalogue number- 28908, THERMO Scientific) for $10 \mathrm{~min}$, lysed and sonicated. ChIP samples were reversecrosslinked and DNA was purified using a Qiaquick column (Qiagen). Target sites obtained from ChIP-seq analysis were further validated by quantitative PCR using Power SYBR Green Master Mix (Applied Biosystems).

\section{Sodium butyrate treatment of $P$. falciparum}

Parasites were treated with $10 \mathrm{mM}$ sodium butyrate for 0 , 5, 10, $30 \mathrm{~min}, 1,2,4,8$ and $16 \mathrm{~h}$. Parasites were crosslinked and ChIP was carried out as described above using H3K9ac antibody [18]. Levels of acetylation were compared by quantitative real-time quantitative PCR using Power SYBR Green Master Mix (Applied Biosystems).

\section{Gene ontology analysis}

Gene ontology analysis was performed using MADIBA [68]; an online web interface (http://madiba.bi.up.ac.za/).

\section{Additional files}

Additional file 1: Supplementary Figures (S1-S9). (DOCX $2070 \mathrm{~kb}$ )

Additional file 2: Table S4. List of the enhancer-like elements identified in Plasmodium falciparum. (XLS $71 \mathrm{~kb}$ )

Additional file 3: Table S5. Overlap between New TSS List from Adjalleyet. al., 2016and the enhancer-like elements identified in this study. (XLS $19 \mathrm{~kb})$

Additional file 4: Table S1. Primers used for ChIP-qPCR are provided as a separate excel-file. Table S2. Co-ordinates and primers used for the cloning of Intergenic regions for luciferase reporter assay are provided as a separate excel-file. (XLSX $12 \mathrm{~kb}$ )

Additional file 5: Table S3. Contact information for ring, trophozoite and schizont stages is provided as a separate excel-file. (XLS $11820 \mathrm{~kb}$ )

\section{Abbreviations \\ ChIP Seq: Chromatin immunoprecipitation sequencing; eRNAs: enhancer RNAs; FAIRE Seq: Formaldehyde-assisted isolation of regulatory elements sequencing; HATs: Histone acetyl transferases; HDACs: Histone deacetylases; IEC: Intra-erythrocytic cycle; IRs: Intergenic regions; ncDNA: Non coding DNA; ORFs: Open reading frames; RBCs: Red blood cells; RPKM: Reads per kilobase per million; TSS: Transcription start site; TTS: Transcription termination site}

\section{Acknowledgements}

We thank Bhagyashree Joshi for help with cloning experiment. We thank Prof. Sanjeev Galande for his generous experimental and resource support and Dr. Pradeepa MM for critical reading of the manuscript. The authors

thank Dr. Abhay Jere for his support. 


\section{Funding}

SU is supported by the Unit of Excellence Program of Department of Biotechnology, Government of India, awarded to Sanjeev Galande. This work was supported by grants under Department of Science and Technology (DST)-Innovation in Science Pursuit for Inspired Research (INSPIRE) Faculty Award (IFA-13, LMBM-53) and Department of Biotechnology (DBT)-Innovative Young Biotechnologist Award (IYBA) (BT/08/IYBA/2014-17) from Government of India to KK

\section{Availability of data and materials}

ChiP-sequencing data used in this study are available under the accession numbers GSE63369, GSE23865 and GSE50199 at Gene Expression Omnibus (GEO).

\section{Authors' contributions}

KK analysed ChIP-seq data. SU designed, performed experiments, and analyzed data. MR cultured $P$. falciparum and performed experiments. KK and SU wrote the manuscript. SV and KA analysed Hi-C data. KK planned, coordinated and supervised the project. All authors read and approved the final manuscript.

\section{Ethics approval and consent to participate}

Not applicable.

\section{Consent for publication}

Not applicable.

\section{Competing interests}

The authors declare that they have no competing interests.

\section{Publisher's Note}

Springer Nature remains neutral with regard to jurisdictional claims in published maps and institutional affiliations.

\section{Author details}

'Department of Biology, Indian Institute of Science Education and Research, Pashan, Pune 411008, India. 'Labs, Persistent Systems Limited, Pingala Aryabhata, Erandwane, Pune 411004, India.

\section{Received: 6 January 2017 Accepted: 11 August 2017}

Published online: 23 August 2017

\section{References}

1. World Malaria Report 2014. Human Rights Documents online. Available from: http://dx.doi.org/10.1163/2210-7975_hrd-9841-2014002.

2. Gardner MJ, Hall N, Fung E, White O, Berriman M, Hyman RW, et al. Genome sequence of the human malaria parasite plasmodium falciparum. Nature 2002;419:498-511. Available from: http://dx.doi.org/10.1038/nature01097.

3. Siegel TN, Hon C-C, Zhang Q, Lopez-Rubio J-J, Scheidig-Benatar C, Martins RM, et al. Strand-specific RNA-Seq reveals widespread and developmentally regulated transcription of natural antisense transcripts in plasmodium falciparum. BMC Genomics. 2014;15:150. Available from: http://dx.doi.org/10. 1186/1471-2164-15-150.

4. Otto TD, Wilinski D, Assefa S, Keane TM, Sarry LR, Böhme U, et al. New insights into the blood-stage transcriptome of plasmodium falciparum using RNA-Seq. Mol Microbiol. 2010;76:12-24. Available from: http://dx.doi. org/10.1111/j.1365-2958.2009.07026.x

5. Brick K, Watanabe J, Pizzi E. Core promoters are predicted by their distinct physicochemical properties in the genome of plasmodium falciparum. Genome Biol. 2008;9:R178. Available from: http://dx.doi.org/10.1186/gb2008-9-12-r178.

6. Ruvalcaba-Salazar OK, del Carmen R-EM, Montiel-Condado D, Recillas-Targa F, Vargas M, Hernández-Rivas R. Recombinant and native plasmodium falciparum TATA-binding-protein binds to a specific TATA box element in promoter regions. Mol Biochem Parasitol. 2005;140:183-96. Available from: http://dx.doi.org/10.1016/j.molbiopara.2005.01.002.

7. Avraham I, Schreier J, Dzikowski R. Insulator-like pairing elements regulate silencing and mutually exclusive expression in the malaria parasite plasmodium falciparum. Proc Natl Acad Sci. 2012;109:E3678-86. Available from: http://dx.doi.org/10.1073/pnas.1214572109
8. Russell K, Emes R, Horrocks P. Triaging informative cis-regulatory elements for the combinatorial control of temporal gene expression during plasmodium falciparum intraerythrocytic development. Parasit Vectors. 2015 8:81. Available from: http://dx.doi.org/10.1186/s13071-015-0701-0.

9. Militello KT, Dodge M, Bethke L, Wirth DF. Identification of regulatory elements in the plasmodium falciparum genome. Mol Biochem Parasitol. 2004;134:75-88. Available from: https://www.ncbi.nlm.nih.gov/pubmed/ 14747145

10. Balaji S. Discovery of the principal specific transcription factors of Apicomplexa and their implication for the evolution of the AP2-integrase DNA binding domains. Nucleic Acids Res. 2005;33:3994-4006. Available from: http://dx.doi.org/10.1093/nar/gki709.

11. Briquet $\mathrm{S}$, Boschet $\mathrm{C}$, Gissot M, Tissandie E, Sevilla E, Franetich J-F, et al. High-mobility-group box nuclear factors of plasmodium falciparum. Eukaryot Cell. 2006:5:672-82. Available from: http://dx.doi.org/10.1128/ec.5.4. 672-682.2006

12. Boschet C, Gissot M, Briquet S, Hamid Z, Claudel-Renard C, Vaquero C. Characterization of PfMyb1 transcription factor during erythrocytic development of 3D7 and F12 plasmodium falciparum clones. Mol Biochem Parasitol. 2004;138:159-63. Available from: http://dx.doi.org/10.1016/j. molbiopara.2004.07.011.

13. Flueck C, Bartfai R, Niederwieser I, Witmer K, Alako BTF, Moes S, et al. A major role for the plasmodium falciparum ApiAP2 protein PfSIP2 in chromosome end biology. PLoS Pathog. 2010;6:e1000784. Available from: http://dx.doi.org/10.1371/journal.ppat.1000784

14. Ong C-T, Corces VG. Enhancer function: new insights into the regulation of tissue-specific gene expression. Nat Rev Genet. 2011;12:283-93. Available from: http://dx.doi.org/10.1038/nrg2957.

15. López-Estraño C, Gopalakrishnan AM, Semblat J-P, Ross Fergus M, Mazier D, Haldar K. An enhancer-like region regulates hrp3 promoter stage-specific gene expression in the human malaria parasite plasmodium falciparum. Biochim Biophys Acta. 2007;1769:506-13. Available from: http://dx.doi.org/ 10.1016/j.bbaexp.2007.04.009.

16. Komaki-Yasuda K, Okuwaki M, Kano S, Nagata K, Kawazu S-I. 5' sequenceand chromatin modification-dependent gene expression in plasmodium falciparum erythrocytic stage. Mol Biochem Parasitol. 2008;162:40-51. Available from: http://dx.doi.org/10.1016/j.molbiopara.2008.07.002

17. Horrocks $P$, Wong $E$, Russell $K$, Emes RD. Control of gene expression in plasmodium falciparum - ten years on. Mol Biochem Parasitol. 2009;164:925. Available from: http://dx.doi.org/10.1016/j.molbiopara.2008.11.010.

18. Karmodiya K, Pradhan SJ, Joshi B, Jangid R, Reddy PC, Galande S. A comprehensive epigenome map of plasmodium falciparum reveals unique mechanisms of transcriptional regulation and identifies $\mathrm{H} 3 \mathrm{~K} 36 \mathrm{me} 2 \mathrm{as}$ a global mark of gene suppression. Epigenetics Chromatin. 2015;8:32. Available from: http://dx.doi.org/10.1186/s13072-015-0029-1.

19. Rawat M, Bhosale MA, Karmodiya K. Plasmodium falciparum epigenome: a distinct dynamic epigenetic regulation of gene expression. Genomics Data. 2016;7:79-81. Available from: http://dx.doi.org/10.1016/j.gdata.2015.11.026.

20. Salcedo-Amaya AM, van Driel MA, Alako BT, Trelle MB, van den Elzen AMG, Cohen AM, et al. Dynamic histone $\mathrm{H} 3$ epigenome marking during the intraerythrocytic cycle of plasmodium falciparum. Proc Natl Acad Sci U S A. 2009;106:9655-60. Available from: http://dx.doi.org/10.1073/pnas. 0902515106.

21. Russell K, Hasenkamp S, Emes R, Horrocks P. Analysis of the spatial and temporal arrangement of transcripts over intergenic regions in the human malarial parasite plasmodium falciparum. BMC Genomics. 2013;14:267. Available from: http://dx.doi.org/10.1186/1471-2164-14-267.

22. Davie JR, Moniwa M. Control of chromatin remodeling. Crit Rev Eukaryot Gene Expr. 2000;10:24. Available from: http://dx.doi.org/10.1615/ critreveukargeneexpr.v10.i34.70.

23. Merrick CJ, Duraisingh MT. Plasmodium falciparum Sir2: an unusual sirtuin with dual histone deacetylase and ADP-ribosyltransferase activity. Eukaryot Cell. 2007;6:2081-91. Available from: http://dx.doi.org/10.1128/EC.00114-07.

24. Hon GC, Hawkins RD, Ren B. Predictive chromatin signatures in the mammalian genome. Hum Mol Genet. 2009;18:R195-201. Available from: http://dx.doi.org/10.1093/hmg/ddp409.

25. ENCODE Project Consortium. An integrated encyclopedia of DNA elements in the human genome. Nature. 2012;489:57-74. Available from: http://dx.doi. org/10.1038/nature11247.

26. Pekowska A, Benoukraf T, Zacarias-Cabeza J, Belhocine M, Koch F, Holota H, et al. H3K4 tri-methylation provides an epigenetic signature of active 
enhancers. EMBO J. 2011;30:4198-210. Available from: http://dx.doi.org/10. 1038/emboj.2011.295.

27. Djebali S, Davis CA, Merkel A, Dobin A, Lassmann T, Mortazavi A, et al. Landscape of transcription in human cells. Nature. 2012;489:101-8. Available from: http://dx.doi.org/10.1038/nature11233.

28. Heintzman ND, Stuart RK, Hon G, Fu Y, Ching CW, Hawkins RD, et al. Distinct and predictive chromatin signatures of transcriptional promoters and enhancers in the human genome. Nat Genet. 2007;39:311-8. Available from: http://dx.doi.org/10.1038/ng1966.

29. Karmodiya K, Krebs AR, Oulad-Abdelghani M, Kimura H, Tora L. H3K9 and H3K14 acetylation co-occur at many gene regulatory elements, while $\mathrm{H} 3 \mathrm{~K} 14 \mathrm{ac}$ marks a subset of inactive inducible promoters in mouse embryonic stem cells. BMC Genomics. 2012;13:424. Available from: http://dx. doi.org/10.1186/1471-2164-13-424.

30. Roh T-Y, Wei G, Farrell CM, Zhao K. Genome-wide prediction of conserved and nonconserved enhancers by histone acetylation patterns. Genome Res. 2007:17:74-81. Available from: http://dx.doi.org/10.1101/gr.5767907.

31. Ponts N, Harris EY, Prudhomme J, Wick I, Eckhardt-Ludka C, Hicks GR, et al. Nucleosome landscape and control of transcription in the human malaria parasite. Genome Res. 2010;20:228-38. Available from: http://dx.doi.org/10. 1101/gr.101063.109.

32. Crabb BS, Triglia T, Waterkeyn JG, Cowman AF. Stable transgene expression in plasmodium falciparum. Mol Biochem Parasitol. 1997;90:131-44. Available from: http://dx.doi.org/10.1016/s0166-6851(97)00143-6.

33. Deitsch K, Driskill C, Wellems T. Transformation of malaria parasites by the spontaneous uptake and expression of DNA from human erythrocytes. Nucleic Acids Res. 2001;29:850-3. Available from: https:/www.ncbi.nlm.nih. gov/pubmed/11160909

34. Koch F, Fenouil R, Gut M, Cauchy P, Albert TK, Zacarias-Cabeza J, et al. Transcription initiation platforms and GTF recruitment at tissue-specific enhancers and promoters. Nat Struct Mol Biol. 2011;18:956-63. Available from: http://dx.doi.org/10.1038/nsmb.2085.

35. De Santa F, Barozzi I, Mietton F, Ghisletti S, Polletti S, Tusi BK, et al. A large fraction of extragenic RNA pol II transcription sites overlap enhancers. PLoS Biol. 2010;8:e1000384. Available from: http://dx.doi.org/10.1371/journal.pbio. 1000384 .

36. Khalil AM, Guttman M, Huarte M, Garber M, Raj A, Rivea Morales D, et al. Many human large intergenic noncoding RNAs associate with chromatinmodifying complexes and affect gene expression. Proc Natl Acad Sci U S A. 2009;106:11667-72. Available from: http://dx.doi.org/10.1073/pnas. 0904715106.

37. Adjalley SH, Chabbert CD, Klaus B, Pelechano V, Steinmetz LM. Landscape and dynamics of transcription initiation in the malaria parasite plasmodium falciparum. Cell Rep. 2016;14:2463-75. Available from: http://dx.doi.org/10. 1016/j.celrep.2016.02.025.

38. Sexton T, Cavalli G. The role of chromosome domains in shaping the functional genome. Cell. 2015;160:1049-59. Available from: http://dx.doi.org/ 10.1016/j.cell.2015.02.040

39. Ay F, Bunnik EM, Varoquaux N, Bol SM, Prudhomme J, Vert J-P, et al. Threedimensional modeling of the P. Falciparum genome during the erythrocytic cycle reveals a strong connection between genome architecture and gene expression. Genome Res. 2014;24:974-88. Available from: http://dx.doi.org/ 10.1101/gr.169417.113.

40. Lemieux JE, Kyes SA, Otto TD, Feller Al, Eastman RT, Pinches RA, et al. Genome-wide profiling of chromosome interactions in plasmodium falciparum characterizes nuclear architecture and reconfigurations associated with antigenic variation. Mol Microbiol. 2013;90:519-37. Available from: http://dx.doi.org/10.1111/mmi.12381.

41. Ay F, Bunnik EM, Varoquaux N, Vert J-P, Noble WS, Le Roch KG. Multiple dimensions of epigenetic gene regulation in the malaria parasite plasmodium falciparum: gene regulation via histone modifications, nucleosome positioning and nuclear architecture in P. Falciparum. BioEssays. 2015;37:182-94. Available from: http://dx.doi.org/10.1002/bies. 201400145.

42. Moraes CB, Dorval T, Contreras-Dominguez M, Dossin Fde M, MAE H, Genovesio A, et al. Transcription sites are developmentally regulated during the asexual cycle of plasmodium falciparum. PLoS One. 2013;8:e55539. Available from: http://dx.doi.org/10.1371/journal.pone.0055539.

43. Westenberger SJ, Cui L, Dharia N, Winzeler E, Cui L. Genome-wide nucleosome mapping of plasmodium falciparum reveals histone-rich coding and histone-poor intergenic regions and chromatin remodeling of core and subtelomeric genes. BMC Genomics. 2009;10:610. Available from: http://dx.doi.org/10.1186/1471-2164-10-610.

44. Plank JL, Dean A. Enhancer function: mechanistic and genome-wide insights come together. Mol Cell. 2014;55:5-14. Available from: http://dx.doi. org/10.1016/.jmolcel.2014.06.015

45. Mousavi K, Zare H, Dell'orso S, Grontved L, Gutierrez-Cruz G, Derfoul A, et al. eRNAs promote transcription by establishing chromatin accessibility at defined genomic loci. Mol Cell. 2013;51:606-17. Available from: http://dx.doi. org/10.1016/j.molcel.2013.07.022.

46. Creyghton MP, Cheng AW, Welstead GG, Kooistra T, Carey BW, Steine EJ, et al. Histone H3K27ac separates active from poised enhancers and predicts developmental state. Proc Natl Acad Sci U S A. 2010;107:21931-6. Available from: http://dx.doi.org/10.1073/pnas.1016071107.

47. Shlyueva D, Stampfel G, Stark A. Transcriptional enhancers: from properties to genome-wide predictions. Nat Rev Genet. 2014;15:272-86. Available from: http://dx.doi.org/10.1038/nrg3682.

48. Trelle MB, Salcedo-Amaya AM, Cohen AM, Stunnenberg HG, Jensen ON. Global histone analysis by mass spectrometry reveals a high content of acetylated lysine residues in the malaria parasite plasmodium falciparum. J Proteome Res. 2009;8:3439-50. Available from: http://dx.doi.org/10.1021/ pr9000898.

49. Wang Z, Zang C, Rosenfeld JA, Schones DE, Barski A, Cuddapah S, et al. Combinatorial patterns of histone acetylations and methylations in the human genome. Nat Genet. 2008;40:897-903. Available from: http://dx.doi. org/10.1038/ng.154.

50. Zhu Y, Sun L, Chen Z, Whitaker JW, Wang T, Wang W. Predicting enhancer transcription and activity from chromatin modifications. Nucleic Acids Res. 2013;41:10032-43. Available from: http://dx.doi.org/10.1093/nar/gkt826.

51. Rada-lglesias A, Bajpai R, Swigut T, Brugmann SA, Flynn RA, Wysocka J. A unique chromatin signature uncovers early developmental enhancers in humans. Nature. 2011;470:279-83. Available from: http://dx.doi.org/10.1038/ nature09692.

52. Melgar MF, Collins FS, Sethupathy P. Discovery of active enhancers through bidirectional expression of short transcripts. Genome Biol. 2011;12:R113. Available from: http://dx.doi.org/10.1186/gb-2011-12-11-r113.

53. Ceballos-Chávez M, Subtil-Rodríguez A, Giannopoulou EG, Soronellas D, Vázquez-Chávez E, Vicent GP, et al. The chromatin remodeler CHD8 is required for activation of progesterone receptor-dependent enhancers. PLoS Genet. 2015;11:e1005174. Available from: http://dx.doi.org/10.1371/ journal.pgen.1005174.

54. Alexander JM, Hota SK, He D, Thomas S, Ho L, Pennacchio LA, et al. Brg1 modulates enhancer activation in mesoderm lineage commitment. Development. 2015;142:1418-30. Available from: http://dx.doi.org/10.1242/ dev.109496

55. Lopez-Rubio J-J, Mancio-Silva L, Scherf A. Genome-wide analysis of heterochromatin associates clonally variant gene regulation with perinuclear repressive centers in malaria parasites. Cell Host Microbe. 2009;5: 179-90. Available from: http://dx.doi.org/10.1016/j.chom.2008.12.012.

56. Ralph SA, Scheidig-Benatar C, Scherf A. Antigenic variation in plasmodium falciparum is associated with movement of var loci between subnuclear locations. Proc Natl Acad Sci U S A. 2005;102:5414-9. Available from: http:// dx.doi.org/10.1073/pnas.0408883102

57. Lindner SE, De Silva EK, Keck JL, Llinás M. Structural determinants of DNA binding by a P. Falciparum ApiAP2 transcriptional regulator. J Mol Biol. 2010;395:558-67. Available from: http://dx.doi.org/10.1016/j.jmb.2009.11.004

58. Cai H, Hong C, Gu J, Lilburn TG, Kuang R, Wang Y. Module-based subnetwork alignments reveal novel transcriptional regulators in malaria parasite plasmodium falciparum. BMC Syst Biol. 2012;6(Suppl 3):S5. Available from: http://dx.doi.org/10.1186/1752-0509-6-S3-S5

59. Campbell TL, De Silva EK, Olszewski KL, Elemento O, Llinás M. Identification and genome-wide prediction of DNA binding specificities for the ApiAP2 family of regulators from the malaria parasite. PLoS Pathog. 2010;6: e1001165. Available from: http://dx.doi.org/10.1371/journal.ppat.1001165.

60. Painter HJ, Campbell TL, Llinás M. The Apicomplexan AP2 family: integral factors regulating plasmodium development. Mol Biochem Parasitol. 2011; 176:1-7. Available from: http://dx.doi.org/10.1016/.molbiopara.2010.11.014.

61. Calo E, Wysocka J. Modification of enhancer chromatin: what, how, and why? Mol Cell. 2013;49:825-37. Available from: http://dx.doi.org/10.1016/j. molcel.2013.01.038.

62. Merika M, Thanos D. Enhanceosomes. Curr Opin Genet Dev. 2001;11:205-8. Available from: http://dx.doi.org/10.1016/s0959-437x(00)00180-5. 
63. Radfar A, Méndez D, Moneriz C, Linares M, Marín-García P, Puyet A, et al. Synchronous culture of plasmodium falciparum at high parasitemia levels. Nat Protoc. 2009;4:1899-915. Available from: http://dx.doi.org/10.1038/nprot. 2009.198.

64. Bártfai R, Hoeijmakers WAM, Salcedo-Amaya AM, Smits AH, Janssen-Megens E, Kaan A, et al. H2A.Z demarcates Intergenic regions of the plasmodium falciparum Epigenome that are dynamically marked by H3K9ac and H3K4me3. PLoS Pathog. 2010;6:e1001223. Available from: http://dx.doi.org/ 10.1371/journal.ppat.1001223.

65. Jiang L, Mu J, Zhang Q, Ni T, Srinivasan P, Rayavara K, et al. PfSETvs methylation of histone H3K36 represses virulence genes in plasmodium falciparum. Nature. 2013;499:223-7. Available from: http://dx.doi.org/10. 1038/nature12361.

66. Ye T, Krebs AR, Choukrallah M-A, Keime C, Plewniak F, Davidson I, et al. seqMINER: an integrated ChIP-seq data interpretation platform. Nucleic Acids Res. 2011;39:e35. Available from: http://dx.doi.org/10.1093/nar/ gkq1287.

67. Krebs AR, Karmodiya K, Lindahl-Allen M, Struhl K, Tora L. SAGA and ATAC Histone acetyl Transferase complexes regulate distinct sets of genes and ATAC defines a class of p300-independent enhancers. Mol Cell. 2011;44: 410-23. Available from: http://dx.doi.org/10.1016/j.molcel.2011.08.037.

68. Law PJ, Claudel-Renard C, Joubert F, Louw Al, Berger DK. MADIBA: a web server toolkit for biological interpretation of plasmodium and plant gene clusters. BMC Genomics. 2008;9:105. Available from: http://dx.doi.org/10. 1186/1471-2164-9-105.

\section{Submit your next manuscript to BioMed Central and we will help you at every step:}

- We accept pre-submission inquiries

- Our selector tool helps you to find the most relevant journal

- We provide round the clock customer support

- Convenient online submission

- Thorough peer review

- Inclusion in PubMed and all major indexing services

- Maximum visibility for your research

Submit your manuscript at www.biomedcentral.com/submit 